\title{
Role of Ultrasonic Elastography in the Evaluation of Fibrosis in Children with Chronic Liver Disease in Comparison to Liver Biopsy
}

\author{
Soumaya E. Hadhod*1, Mohamed Z.A. Mourad ${ }^{2}$, Ashraf M. Radwan', Omar A.A. Ahmed ${ }^{1}$ \\ Departments of ${ }^{1}$ Pediatrics and ${ }^{2}$ Diagnostic Radiology, Faculty of Medicine, Sohag University, Egypt \\ *Corresponding author: Soumaya E. Hadhod, Mobile: (+20)1064463697, E-Mail: s_hadhood55@yahoo.com
}

\begin{abstract}
Background: Chronic liver illnesses can be caused by a variety of factors, including metabolic, genetic, drug-induced, inflammatory, structural, dietary, and immunological factors. Even with Computed Tomography (CT) scans or Magnetic Resonance Imaging (MRI), liver fibrosis makes diagnostic imaging difficult in chronic liver injury. Percutaneous liver biopsy is the gold standard for diagnosing, grading, and monitoring liver fibrosis in children, but it is painful, invasive, requires heavy sedation, and has numerous complications such as bleeding.

Objectives: The aim of the current work was to assess and measure liver stiffness in children with chronic liver disease using real-time elastography (RTE), compare the results to histopathological findings obtained after performing a percutaneous liver biopsy, and investigate the availability of noninvasive safe liver elastography as a future replacement for this invasive tool in the evaluation and grading of liver fibrosis.

Patients and methods: This hospital-based cross-sectional comparative study included a total of 30 individuals with chronic liver disease of various etiologies with age ranged from 4-16 years, attending at Department of Pediatric, Sohag University Hospitals. This study was conducted between January 2017 to December 2019. A control group of ten healthy people was also included in the investigation.

Results: The degree of liver stiffness measured by the last ultrasonic elastography was greater in the patient group than in the control group, with a statistically significant difference between the two groups. Except for F0-F1 and F3F4, there was a substantial relationship between fibrosis stage and liver stiffness assessment by sonoelastography, where the difference was significant between every two individual groups. There was a strong link between biopsy diagnosis and liver stiffness measurement.
\end{abstract}

Conclusion: It could be concluded that in adults, ultrasonic elastography is a well-established approach for assessing liver fibrosis. The utility of elastography in the follow-up of children with chronic liver disease might be increased.

Keywords: Fibrosis, Liver biopsy, Ultrasonic elastography, Chronic liver disease

\section{INTRODUCTION}

Chronic liver illnesses are defined by six months of clinical or biochemical evidence of hepatic dysfunction. In Egypt, liver problems are a significant source of illness and mortality. When there are clinical and biochemical signs of liver failure, chronic liver disease can be compensated or decompensated ${ }^{(\mathbf{1})}$.

Fibrosis is a complicated condition that involves both parenchymal and non-parenchymal liver cells, as well as invading immune cells. The activated hepatic stellate cell is the most important fibrogenic effector cell type in the liver (HSC). The characteristics of HSC activation and phenotypic transition into myofibroblasts, as well as their pro-fibrogenic activity, have been widely defined, and they serve as a critical foundation for understanding hepatic fibrogenesis ${ }^{(2)}$.

The criteria standard for determining the genesis and degree of liver disease is still liver biopsy. Although liver biopsy is typically safe and is now regarded as the gold standard for assessing hepatic inflammation and fibrosis, it is not without risk. Sampling errors, uncommon complications, and substantial patient anxiety have all been reported. These variables have sparked a great deal of interest in the development of noninvasive hepatic fibrosis diagnostics ${ }^{(3)}$.

The elastography technique works by applying small external tissue compression to the structures being studied, causing strain (displacement) inside the tissue, and then calculating the strain profile along the compression axis. The comparison of signals obtained before and after tissue movement is the basis of ultrasonic elastography. There have been several ultrasonic elastographic methods developed ${ }^{(4)}$.

In 34 children, Schenk et al. ${ }^{(5)}$ compared the usefulness of ultrasonic elastography to liver biopsy in pediatric liver disorders. They concluded that RTE characteristics can help differentiate between high and low fibrosis, however, their link with the fibrosis stage is only mild. RTE appears to be a viable approach for assessing liver fibrosis in youngsters.

The aim of the current work was to assess and measure liver stiffness in children with chronic liver disease using real-time elastography (RTE), compare the results to histopathological findings obtained after performing a percutaneous liver biopsy, and investigate the availability of noninvasive safe liver elastography as a future replacement for this invasive tool in the evaluation and grading of liver fibrosis.

\section{PATIENTS AND METHODS}

This hospital-based cross-sectional comparative study included a total of 30 individuals with chronic liver disease of various etiologies with age ranged from 416 years, attending at Department of Pediatric, Sohag University Hospitals. This study was conducted 
between January 2017 to December 2019). A control group of ten healthy people was also included in the investigation.

Inclusion criteria: All kids suspected of having chronic liver disease aged 4 to 16 years were given a thorough history, a thorough clinical examination, and several tests, as well as a percutaneous liver biopsy and RTE.

Exclusion criteria: Patients or caregivers who refuse to grant written informed permission to participate in the research, and patients with decompensated liver illness or ascitis, as fluid, interfere with wave propagation.

\section{All patients were subjected to:}

- Recording history.

- Exams on a national and local level.

- Anthropometric evaluations (weight, height, and BMI).

- When autoimmune or storage illnesses are suspected, laboratory testing of liver function tests, coagulation profile, and serology for hepatitis B and hepatitis $\mathrm{C}$ serology were performed.

- Ultrasound of the abdomen.

- Biopsy and histological evaluation of the liver.

- Ultrasonic elastography.

\section{Ethical consent:}

The study was approved by the Ethical Committee, Faculty of Medicine, Sohag University. Informed written consent was obtained from parents of all children participants before recruitment in the study, after explaining the objectives of the work. Confidentiality was guaranteed on handling the data base. This work has been carried out in accordance with The Code of Ethics of the World Medical Association (Declaration of Helsinki) for studies involving humans.

\section{Statistical analysis}

Epi-Info version 6 and SPP for Windows version 8 were used to enter, validate, and analyze data. The arithmetic means standard deviation, median, Mann Whitney- $U$ test, the validity of screening test, $t-$ test, and Chi-squared test were used to summarise the data. The correlation coefficient " $r$ " was used to calculate the correlation between variables. This test determines whether or not a change in one variable was accompanied by a change in the other. The threshold of significance for all of the abovementioned statistical tests is set at $5 \%$. P value $<0.05$ was considered significant.

\section{RESULTS}

Table 1 displays the demographics of the 40 children that were examined. The children's average age was 10.23 years in the case group and 10.75 years in the control group, with a range of 5.5 to 15 years.
The majority of the patients in the case group (53.3\%) were females, with the remaining instances being men $(46.7 \%)$. In the control group, however, 50 percent of the participants were men and 50 percent were females.

The degree of liver stiffness measured by ultrasonic elastography was higher in the patient group $(24.84 \pm 11.57)$ than in the control group $(7.5 \pm 2.255)$, with a statistically significant difference between the two groups $(\mathrm{p}<0.001)$. (Table 2$)$.

Table (3) reveales a substantial relationship between fibrosis stage and ultrasonic elastography measurements of liver stiffness, with significant differences between the two groups except for F0-F1 and F3-F4.

The best cutoff for measuring liver stiffness by ultrasonic elastography in the diagnosis of $\mathrm{F} 1$ was $\geq 10.5 \mathrm{KPa}$, which had an area under the curve of 0.975 , a sensitivity of 87.5 percent, a specificity of 90 percent, a positive predictive value of 87.5 percent, a negative predictive value of 90 percent, and an accuracy of 88.9 percent $(\mathrm{p}<0.001)$ (Table 4).

With an area under the curve of 0.942 , the sensitivity of 75 percent, specificity of 84.2 percent, the positive predictive value of 50 percent, the negative predictive value of 94.1 percent, and accuracy of 82.6 percent $(p<0.05)$, the best cutoff for liver stiffness measurement by ultrasonic elastography in the diagnosis of autoimmune hepatitis was $20 \mathrm{KPa}$ (Table 5).

The optimum cutoff for liver stiffness assessment by ultrasonic elastography in the diagnosis of $\mathrm{F} 2$ was $\geq 16.5 \mathrm{KPa}$, with an area under the curve of $0.983,100 \%$ sensitivity, 94.7 percent specificity, $80 \%$ positive predictive value, $100 \%$ negative predictive value, and 95.6 percent accuracy $(\mathrm{p}<0.001)$ (Table 6).

The best cutoff for measuring liver stiffness by ultrasonic elastography in the diagnosis of $\mathrm{F} 3$ was $\geq 21$ $\mathrm{KPa}$, with an area under the curve of 0.978 , a sensitivity of 85.7 percent, a specificity of 84.6 percent, a positive predictive value of 60 percent, a negative predictive value of 95.7 percent, and an accuracy of 84.8 percent $(\mathrm{p}<0.001)$ (Table 7).

The best cutoff for measuring liver stiffness by ultrasonic elastography in the diagnosis of $\mathrm{F} 3$ was $\geq 34$ $\mathrm{KPa}$, with an area under the curve of 0.924 , the sensitivity of 85.7 percent, specificity of 90.9 percent, the positive predictive value of 66.7 percent, the negative predictive value of $96.8 \%$, and accuracy of 90\% (p<0.001) (Table 8).

The best cutoff for measuring liver stiffness by ultrasonic elastography in the diagnosis of congenital hepatic fibrosis was $\geq 29 \mathrm{KPa}$, with an area under the curve of 0.942 , the sensitivity of $100 \%$, specificity of 90.9 percent, the positive predictive value of 71.4 percent, the negative predictive value of $100 \%$, and accuracy of 92.6 percent $(p<0.001)$ (Table 9).

There was a link between biopsy diagnosis and liver stiffness measurement (the greatest value was obtained in congenital fibrosis patients) (Table 10). 
Table (1): In terms of demographic traits, there is a comparison between the groups analyzed.

\begin{tabular}{|c|c|c|c|c|}
\hline \multirow[b]{2}{*}{$\begin{array}{l}\text { Demographic } \\
\text { characteristics }\end{array}$} & \multicolumn{2}{|c|}{ Groups } & \multicolumn{2}{|c|}{ Test } \\
\hline & $\begin{array}{c}\text { Case group } \\
\mathbf{N}=\mathbf{3 0}(\%)\end{array}$ & $\begin{array}{c}\text { Control group } \\
\mathrm{N}=10(\%)\end{array}$ & $\chi^{2} / \mathrm{t}$ & p \\
\hline $\begin{array}{l}\text { Gender: } \\
\text { Male } \\
\text { Female }\end{array}$ & $\begin{array}{l}14(46.7) \\
16(53.3) \\
\end{array}$ & $\begin{array}{l}5(50) \\
5(50) \\
\end{array}$ & 0.033 & 0.855 \\
\hline $\begin{array}{l}\text { Age (years): } \\
\text { Mean } \pm \text { SD } \\
\text { Range }\end{array}$ & $\begin{array}{c}10.23 \pm 2.87 \\
5.5-15\end{array}$ & $\begin{array}{c}10.75 \pm 2.76 \\
5.5-15\end{array}$ & -0.497 & 0.622 \\
\hline
\end{tabular}

$\chi^{2}$ Chi square test $t$ independent sample $t$ test

Table (2): Ultrasonic elastography findings were compared between the groups tested.

\begin{tabular}{|c|c|c|c|c|}
\hline \multirow{2}{*}{ Elastography } & \multicolumn{2}{|c|}{ Groups } & \multicolumn{2}{|c|}{ Test } \\
\cline { 2 - 3 } & Case group & Control group & \multirow{2}{*}{$\mathbf{Z}$} & \multirow{2}{*}{$\mathbf{~}$} \\
\cline { 2 - 3 } & $\mathbf{N = 3 0 ( \% )}$ & $\mathbf{N = 1 0}(\mathbf{\%})$ & \multirow{2}{*}{-4.223} & $<0.001^{* *}$ \\
\hline Mean \pm SD & $24.84 \pm 11.57$ & $7.5 \pm 2.55$ & \\
Median (Range) & $23.5(4.5-40.8)$ & $7.5(4-11)$ & & \\
\hline
\end{tabular}

$\mathrm{Z}$ Mann Whitney test ${ }^{* *} \mathrm{p} \leq 0.001$ is statistically highly significant

Table (3): Among the individuals investigated, there was a link between fibrosis stages determined by liver biopsy and liver stiffness measured by elastography.

\begin{tabular}{|c|c|c|c|c|}
\hline & Mean \pm SD & Range & F & p \\
\hline F0 & $6.75 \pm 3.18$ & $4.5-9$ & & \\
\cline { 1 - 3 } F1 & $13.63 \pm 2.13$ & $10-17$ & \multirow{3}{*}{39.51} & $<0.001 * *$ \\
\hline F2 & $21.33 \pm 4.18$ & $15-26$ & & \\
\hline F3 & $32.97 \pm 7.69$ & $20-40$ & & \\
\hline F4 & $37.68 \pm 2.77$ & $33-40.8$ & & \\
\hline
\end{tabular}

F One way ANOVA $\quad * * \mathrm{p} \leq 0.001$ is statistically highly significant

Table (4): When compared to liver biopsy, ultrasonic elastography performed better in diagnosing F1.

\begin{tabular}{|c|c|c|c|c|c|c|c|}
\hline Cutoff & AUC & Sensitivity & Specificity & PPV & NPV & Accuracy & p \\
\hline$\geq 10.5 \mathrm{KPa}$ & 0.975 & 87.5 & 90 & 87.5 & 90 & 88.9 & $0.001^{* *}$ \\
\hline
\end{tabular}

$* * \mathrm{p} \leq 0.001$ is statistically highly significant

Table (5): When compared to liver biopsy, ultrasonic elastography performed better in diagnosing autoimmune hepatitis.

\begin{tabular}{|c|c|c|c|c|c|c|c|}
\hline Cutoff & AUC & Sensitivity & Specificity & PPV & NPV & Accuracy & p \\
\hline$\geq 20 \mathrm{KPa}$ & 0.942 & 75 & 84.2 & 50 & 94.1 & 82.6 & $0.035^{*}$ \\
\hline
\end{tabular}

$* \mathrm{p}<0.05$ is statistically significant

Table (6): When compared to liver biopsy, ultrasonic elastography performed better in diagnosing F2.

\begin{tabular}{|c|c|c|c|c|c|c|c|}
\hline Cutoff & AUC & Sensitivity & Specificity & PPV & NPV & Accuracy & p \\
\hline$\geq 16.5 \mathrm{KPa}$ & 0.983 & 100 & 94.7 & 80 & 100 & 95.6 & $<0.001 * *$ \\
\hline
\end{tabular}

$* * \mathrm{p} \leq 0.001$ is statistically highly significant

Table (7): When compared to liver biopsy, ultrasonic elastography performed better in diagnosing F3.

\begin{tabular}{|c|c|c|c|c|c|c|c|}
\hline Cutoff & AUC & Sensitivity & Specificity & PPV & NPV & Accuracy & p \\
\hline$\geq 21 \mathrm{KPa}$ & 0.978 & 85.7 & 84.6 & 60 & 95.7 & 84.8 & $<0.001^{* *}$ \\
\hline
\end{tabular}

$* * \mathrm{p} \leq 0.001$ is statistically highly significant

Table (8): When compared to liver biopsy, ultrasonic elastography performed better in diagnosing F4.

\begin{tabular}{|c|c|c|c|c|c|c|c|}
\hline Cutoff & AUC & Sensitivity & Specificity & PPV & NPV & Accuracy & p \\
\hline$\geq 34 \mathrm{KPa}$ & 0.924 & 85.7 & 90.9 & 66.7 & 96.8 & 90 & $<0.001^{* *}$ \\
\hline
\end{tabular}

${ }^{* *} \mathrm{p} \leq 0.001$ is statistically highly significant 
Table (9): When compared to liver biopsy, ultrasonic elastography performed better in diagnosing congenital hepatic fibrosis.

\begin{tabular}{|c|c|c|c|c|c|c|c|}
\hline Cutoff & AUC & Sensitivity & Specificity & PPV & NPV & Accuracy & p \\
\hline$\geq 29 \mathrm{KPa}$ & 0.942 & 100 & 90.9 & 71.4 & 100 & 92.6 & $0.001^{* *}$ \\
\hline
\end{tabular}

$* * \mathrm{p} \leq 0.001$ is statistically highly significant

Table (10): The relationship between biopsy diagnosis and liver stiffness measurements

\begin{tabular}{|c|c|c|c|c|c|c|}
\hline 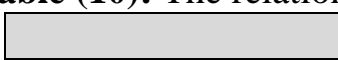 & Mean & SD & Minimum & Maximum & $\mathbf{F}$ & p \\
\hline $\begin{array}{l}\text { Autoimmune } \\
\text { hepatitis }\end{array}$ & 21.75 & 2.87 & 18.00 & 25.00 & \multirow{7}{*}{5.595} & \multirow{7}{*}{$0.001 * *$} \\
\hline Castle man disease & 30.00 & .000 & 30.00 & 30.00 & & \\
\hline Chronic hepatitis & 21.91 & 11.61 & 13.00 & 40.00 & & \\
\hline $\begin{array}{l}\text { Congenital liver } \\
\text { fibrosis }\end{array}$ & $38.77^{\infty}$ & 3.01 & 33.00 & 40.80 & & \\
\hline Fatty liver & 6.75 & 3.18 & 4.50 & 9.00 & & \\
\hline Thalassemia major & 25.00 & 7.07 & 20.00 & 30.00 & & \\
\hline Undiagnosed & 13.00 & 4.24 & 10.00 & 16.00 & & \\
\hline
\end{tabular}

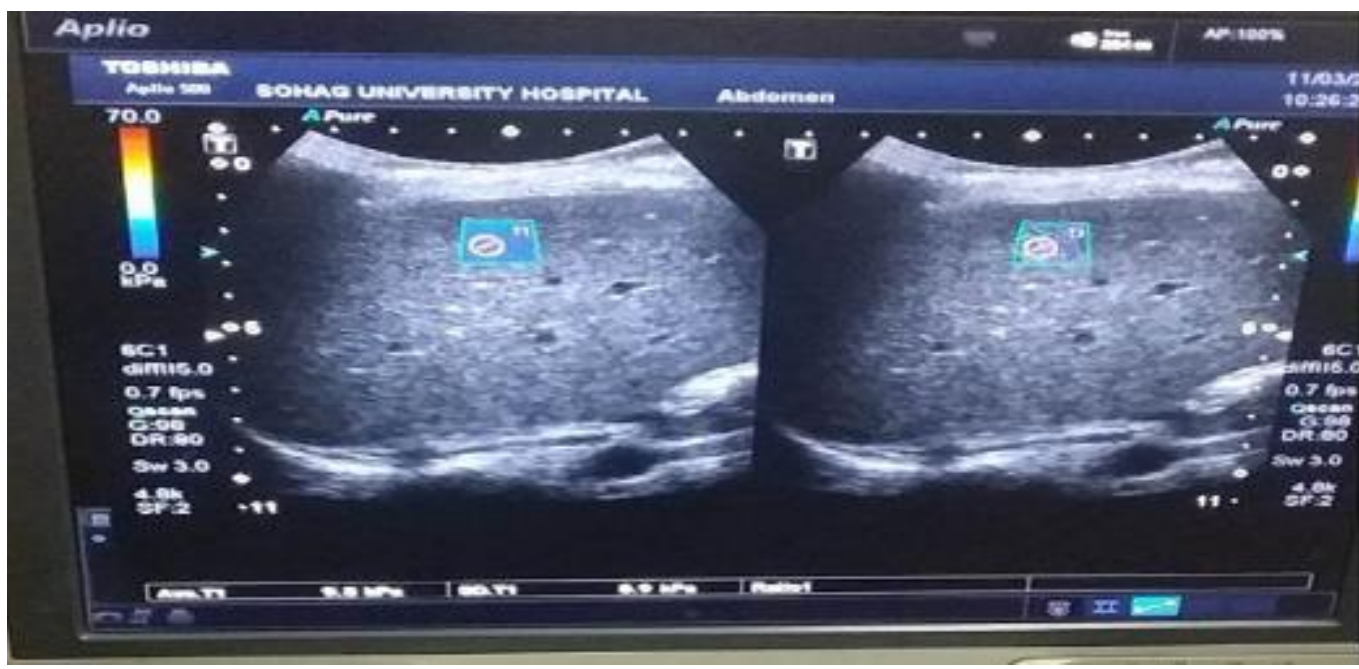

Control: Female patient aged $13 \mathrm{y}$, her weight is $30 \mathrm{~kg}$, and her height is $145 \mathrm{~cm}$, was completely normal clinically and by abdominal ultrasonography selected as a control case and her son elastography measure was $9.8 \mathrm{kph}$.

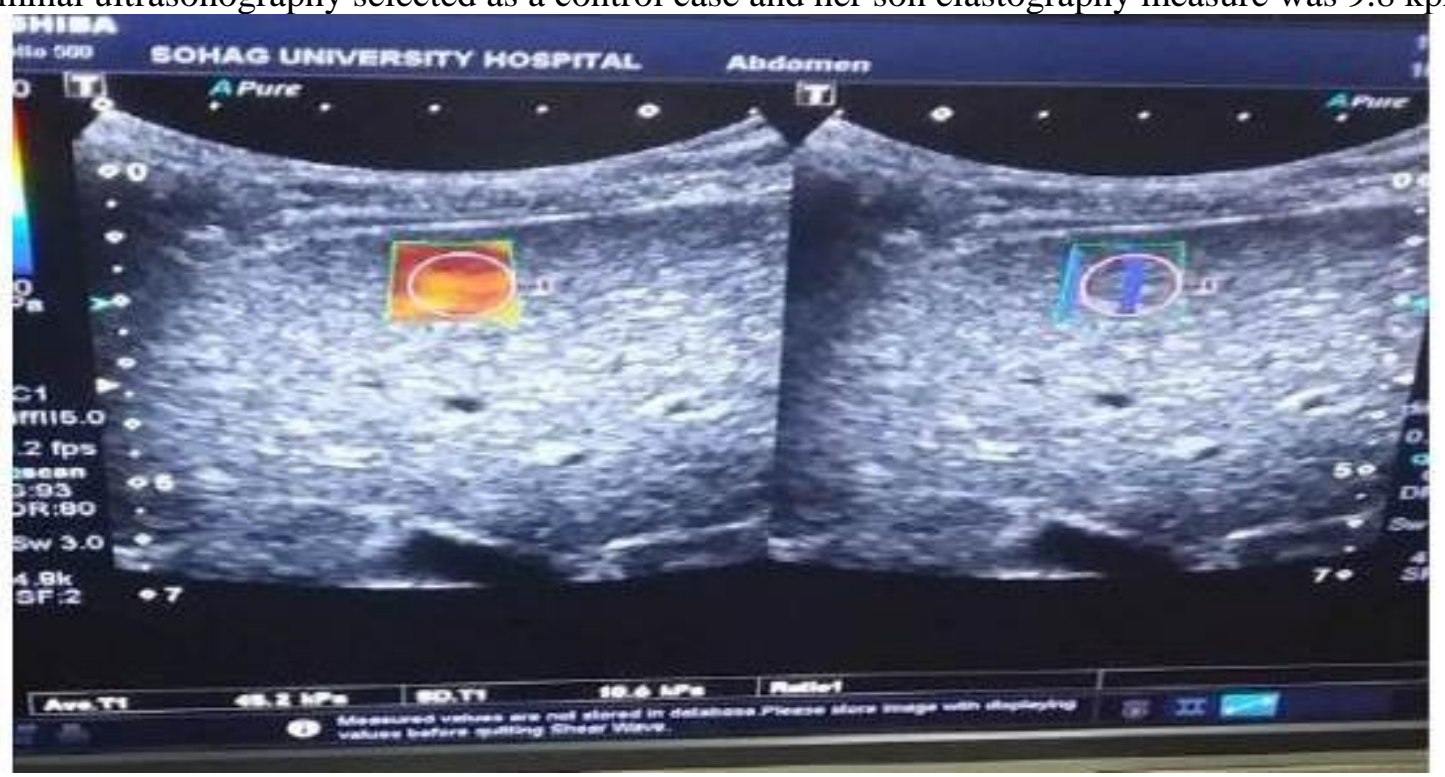

Case 1: Five years old male patient, presented with abdominal distension and yellowish discoloration of sclerae. His clinical examination revealed hepatosplenomegaly, -ve test for ascites. Abdominal ultrasonography revealed liver span $12.5 \mathrm{~cm}$, coarse echo pattern, early hepatorenal collaterals spleen span was $12 \mathrm{~cm}$, mildly enlarged his portal Doppler mild portal by pretension. Liver biopsy and his pathology revealed congenital hepatic fibrosis and his son's elastography measure was 45 kps [Fibrosis (F4)]. 


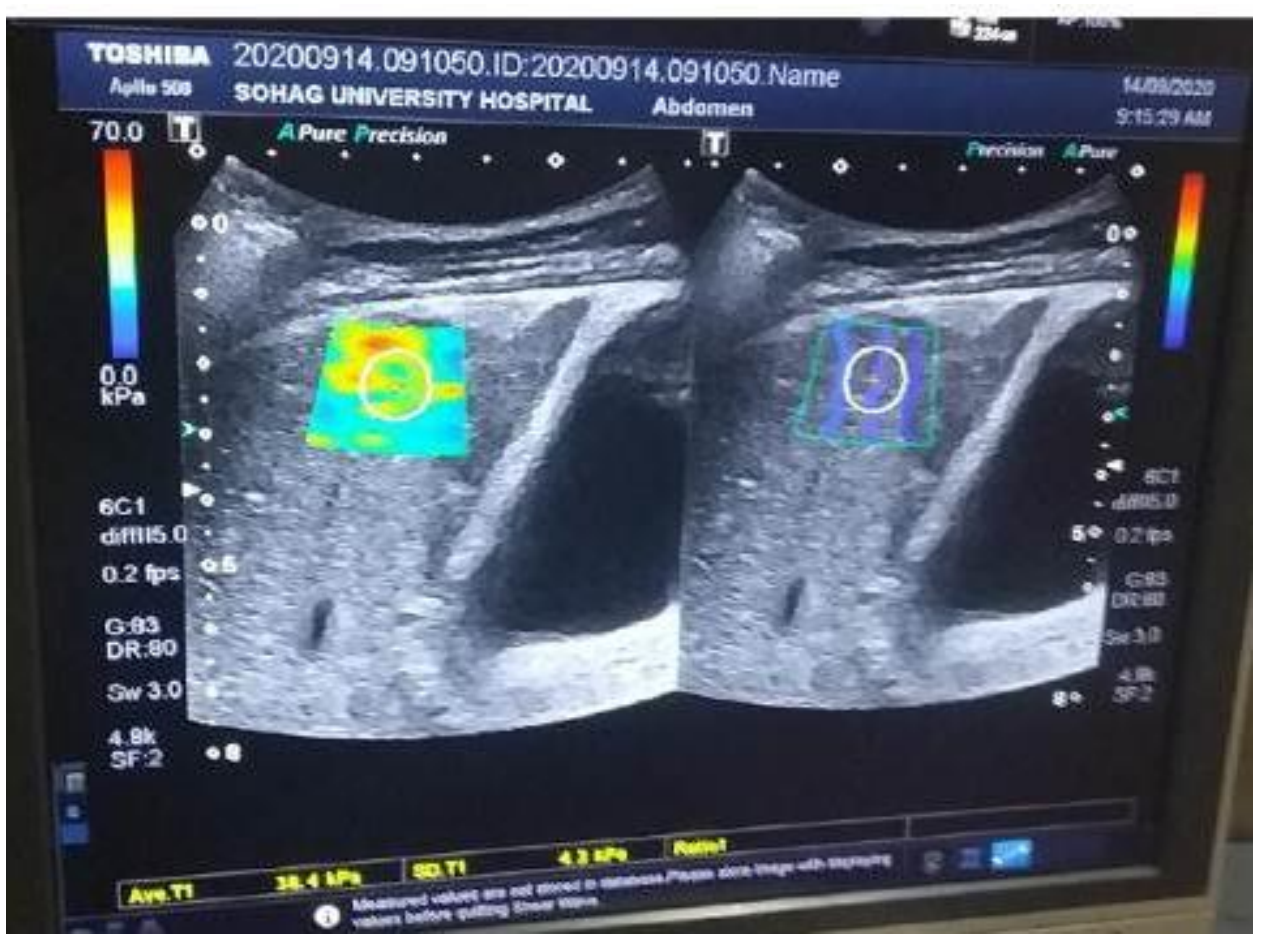

Case 2: Male patient, 16 years old, presented. with Hepatosplenomegaly, by histopathology $\rightarrow$ congenital hepatic fibrosis by sonoelastography $\rightarrow 34 \mathrm{kph}$.

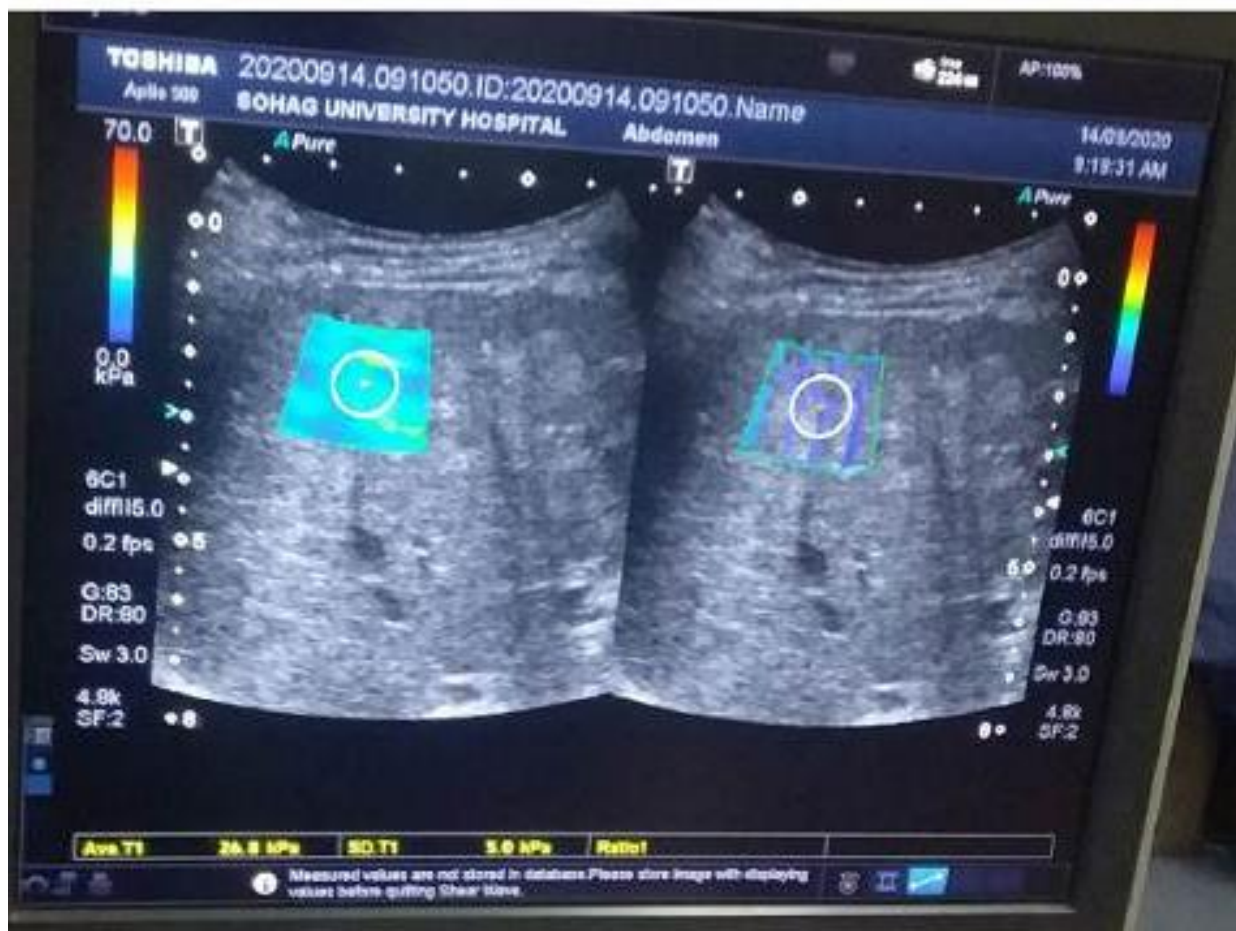

Case 3: Female patient, 8 years presented with a picture of hepatomegaly, her clinicopathological investigations were suggestive of Auto immure hepatitis ( F 2-3). By ultrasonic elastography, measurement was $26.8=$ average.

\section{DISCUSSION}

Our study found that there is no statistically significant difference in age or gender amongst the groups tested. The case group (14 men and 16 females) had a mean age of $10.23 \pm 2.87$ years, while the control group had a mean age of $10.75 \pm 2.76$ years (5 males and 5 females).

Regarding age, Schenk et al. ${ }^{(5)}$ found similar results in a study of 34 children (17 females and 17 boys, mean age $10.53 \pm 5$.66 years), when comparing the usefulness of ultrasonic elastography to liver biopsy in pediatric liver disorders.

Sönmez et al. ${ }^{(6)}$ found similar results when they looked at the diagnostic utility of Share-wave Elastography (SWE) in children with chronic liver disease. A total of 81 individuals with chronic liver disease and 26 healthy controls were included in the research. The patients who took part in the trial had to be at least 17 years old. 
The degree of liver stiffness measured by ultrasonic elastography was substantially greater in the patient group than in the control group.

Park et al. ${ }^{(7)}$ found similar results when they compared MRE and two-dimensional SWE (2DSWE) hepatic stiffness measurements to quantify liver fibrosis. They concluded that MRE, in comparison to 2D-SWE and serum-based indices, maybe a noninvasive and more precise measurement for the assessment of significant fibrosis in treatment $\mathrm{CHB}$ patients with high viral loads but normal or mildly elevated ALT levels who should be considered for antiviral therapy based on the presence of significant fibrosis.

In this study, there was a statistically significant difference between the analyzed groups when measuring the enlarged liver or splenic span. According to Andersen et al. ${ }^{(8)}$, ultrasonography elastography can detect cirrhosis and differentiate healthy from fibrotic liver tissue.

Except for F0-F1 and F3-F4, there was a significant relationship between fibrosis stage and liver stiffness determined by ultrasonic elastography in the current investigation, where the difference was substantial between every two individual groups. This is in agreement with Hanquinet et al. (9), who found that elastography values rise as fibrosis progresses; nevertheless, there are certain difficulties in evaluating the early stages of fibrosis.

RTE measures, according to Schenk et al. ${ }^{(5)}$, allow for the classification of high fibrosis, although their connection with the fibrosis stage is modest. RTE appears to be a viable approach for assessing liver fibrosis in youngsters. Patients with F0 values in the patient group were compared to the values in the control group by Sönmez et al ${ }^{(6)}$. There was no discernible difference between these two groups.

Sönmez et al. ${ }^{(6)}$ noted that because hepatic fibrosis development is a dynamic process and many hepatic fibrosis etiologies are known to persist throughout life, using the Real-time SWE approach instead of recurrent liver biopsies on patients is a lot easier and smarter method. With future investigations, increasing the clinical application of the Real-Time SWE technique may give a chance to avoid needless liver biopsies by evaluating patients more quickly and cost-effectively.

This is the first study to compare the results of the noninvasive assessment with those of liver histology in pediatric patients. The results, however, must be used with caution due to several restrictions. The cross-sectional nature of our investigation constituted a constraint, thus the panel produced should be longitudinally examined and validated in treatment settings of cohorts of patients with various chronic liver illnesses to determine its diagnostic usefulness in specific situations.
TE values varied from 4.3 to $5.0 \mathrm{kPa}$, with the highest limits recorded from 5.63 to $6.5 \mathrm{kPa}$, while pSWE values ranged from 1.07 to $1.19 \mathrm{~m} / \mathrm{s}$, according to Andersen et al. ${ }^{(8)}$ and Pinto et al. ${ }^{(10)}$ who compared elastography to the results of liver biopsies and discovered that stiffness increased as fibrosis progressed. Cirrhosis was appropriately detected by elastography in general, although severe fibrosis was more difficult to assess. The approach was less accurate in distinguishing between nil, mild, and severe fibrosis in children with liver disease. There were no fibrosis-specific cutoffs recommended. The severity of the necroinflammatory response was linked to the stiffness of the liver.

The optimum cutoff for measuring liver stiffness by ultrasonic elastography in diagnosing F1 was $\geq 10.5 \mathrm{KPa}$, with an area under the curve of 0.975 , sensitivity 87.5 percent, specificity 90 percent, positive predictive value 87.5 percent, negative predictive value 90 percent, and accuracy 88.9 percent $(\mathrm{p}<0.001)$.

The best cutoff for measuring liver stiffness by ultrasonic elastography in the diagnosis of autoimmune hepatitis was $\geq 20 \mathrm{KPa}$, with an area under the curve of 0.942 , the sensitivity of $75 \%$, specificity of 84.2 , the positive predictive value of $50 \%$, the negative predictive value of 94.1 , and accuracy of 82.6 percent $(\mathrm{p}<0.05)$.

The optimum cutoff for measuring liver stiffness by ultrasonic elastography in the diagnosis of F2 was $\geq 16.5 \mathrm{KPa}$, with an area under the curve of 0.983 , the sensitivity of $100 \%$, specificity of 94.7 percent, the positive predictive value of $80 \%$, the negative predictive value of $100 \%$, and accuracy of 95.6 percent $(\mathrm{p}<0.001)$.

With an area under the curve of 0.978 , sensitivity 85.7 percent, specificity 84.6 percent, positive predictive value 60 percent, negative predictive value 95.7 percent, and accuracy 84.8 percent $(p<0.001)$, the best cutoff of liver stiffness measurement by ultrasonic elastography in the diagnosis of $\mathrm{F} 3$ was $\geq 21 \mathrm{KPa}(\mathrm{p}<0.001)$. Also, the best cutoff for measuring liver stiffness by ultrasonic elastography in the diagnosis of F3 was $34 \mathrm{KPa}$, with an area under the curve of 0.924 , the sensitivity of 85.7 percent, specificity of 90.9 percent, the positive predictive value of 66.7 percent, the negative predictive value of $96.8 \%$, and accuracy of 90 percent $(\mathrm{p}<0.001)$.

The best cutoff for measuring liver stiffness by ultrasonic elastography in the diagnosis of congenital hepatic fibrosis was $\geq 29 \mathrm{KPa}$, with an area under the curve of 0.942 , the sensitivity of 100 percent, specificity of 90.9 percent, the positive predictive value of 71.4 percent, the negative predictive value of 100 percent, and accuracy of 92.6 percent $(\mathrm{p}<0.001)$, which was similar to Ferraioli et $\boldsymbol{a l} .{ }^{(11)}$. 
Tomato et al. ${ }^{(12)}$ used a liver fibrosis index developed by multiple regression analyses of the different picture components about the METAVIR score to assess ultrasonic elastography in adult patients with hepatitis C (27-30). They discovered a statistically significant rise in the score as liver fibrosis progressed, but no significant association with steatosis grade in adult patients. RTE was also discovered to be a good approach for predicting liver fibrosis. Furthermore, the liver fibrosis index outperformed serum fibrosis indicators in terms of diagnostic accuracy. Tamaki et al. (13) also demonstrated that RTE was effective in HCV patients with or without steatosis. Wang et al. found similar outcomes for adult hepatitis B patients (14).

MEAN, AREA, SD, and 101 adult hepatitis C patients were examined and connected to METAVIR score by Morikawa et al. ${ }^{(15)}$ who found that MEAN dropped with rising METAVIR score while AREA rose. They also discovered a link between TE (measured in $\mathrm{kPa}$ ) and MEAN, as well as TE and AREA.

In a mixed sample population of diverse disorders, including $\mathrm{HCV}, \mathrm{HBV}$, alcoholic liver disease, AIH, NASH/Non-Alcoholic Fatty Liver Disease (NAFLD), and primary biliary cirrhosis, Colombo et al. (16) compared TE, RTE, and ARFI according to METAVIR. They found that as the fibrosis stage progressed, the elasticity index increased, although with a low Spearman correlation value of 0.363 . The association between TE and ARFI was found to be stronger.

In line with our findings, Yada et al. ${ }^{(17)}$ found a strong link between tissue mean elasticity and histological fibrosis grading, particularly in individuals with fibrosis $\geq F 2$. MEAN was investigated by Orlacchio et al. ${ }^{(\mathbf{1 8})}$ in connection to fibrosis score, steatosis score, and the degree of inflammation. Fibrosis was the sole variable that had statistical significance in modifying tissue mean elasticity. Venturi et $\boldsymbol{a l} .^{(\mathbf{1 9})}$ developed a unique histologic scoring method for long-term allograft fibrosis following liver transplantation in children (separate grading of portal, sinusoidal, and centrilobular regions).

In terms of liver biopsy findings, 10 cases had chronic hepatitis, 5 cases had congenital hepatitis and autoimmune hepatitis, 4 patients had fatty liver, 3 patients had thalassemia major, and 2 patients had Castleman disease, while 2 cases had undiscovered illnesses.

Schenk et al. ${ }^{(5)}$ found that hepatopathy of unknown origin $(n=8)$, autoimmune hepatitis $(n=5)$, control after liver transplantation $(\mathrm{n}=5)$, Wilson's disease $(n=4)$, thalassemia $(n=2)$, glycogenosis $(n=$ $2)$, hepatopathy in hereditary fructose intolerance $(\mathrm{n}=$ $1)$, and alpha-1-antitrypsin deficiency $(\mathrm{n}=1)$

\section{CONCLUSION}

- In adults, sonoelastography is a well-established approach for assessing liver fibrosis. Elastography has the potential to improve the utility of ultrasonography in the monitoring of children with chronic liver disease. It should be done for every kid with chronic liver disease at any stage of the disease, on the first encounter, to record the degree of fibrosis and serve as a baseline for patient follow-up.

- Flag with ultrasonic elastography may be effective in detecting the progression of chronic liver disease in children with hepatitis accompanied by high liver enzymes.

- After a persistent virological response to therapy with interferon-based treatments or direct-acting antiviral medicines, liver stiffness improves considerably. Due to the lack of accuracy of cutoffs determined in viremic individuals, liver stiffness cannot be utilized to stage liver fibrosis or rule out cirrhosis.

- Despite decreases in liver stiffness in individuals with advanced illness, screening for hepatocellular carcinoma and portal hypertension should continue.

\section{Financial support and sponsorship: Nil. Conflict of interest: Nil.}

\section{REFERENCES}

1. Shella F (2011): Chronic viral hepatitis in children. Semin. Pediatr. Gastroenterol. Nutr., 2: 9-14.

2. Lee Y, Wallace M, Friedman S (2015): Pathobiology of liver fibrosis: a translational success story. Gut, 64(5): 830-41.

3. Bamber J, Cosgrove D, Dietrich $\mathrm{C}$ et al. (2013): EFSUMB guidelines and recommendations on the clinical uses of ultrasound elastography. Part 1: basic principles and technology. Ultraschall Med., 34: 16984.

4. Ginat D, Destounis S, Barr R et al. (2009): Ultra Sonic Elastography of Breast and Prostate Lesions. RadioGraphics, 29:2007-2016.

5. Schenk J, Selmi B, Flechtenmacher C et al. (2014): ultrasonic Elastography (RTE) for noninvasive evaluation of fibrosis in liver diseases in children in comparison to liver biopsy. J Med Ultrasonics, 41: $455-462$.

6. Sönmez S, Boşat M, Yurtseven N et al. (2019): The role of elastography in the assessment of chronic liver disease in children. Afri Health Sci., 19(3): 2806-2811.

7. Park H, Choe W, Han H et al. (2019): Assessing significant fibrosis using imaging-based elastography in chronic hepatitis B patients: Pilot study. World J Gastroenterol., 25(25): 3256-3267.

8. Andersen S, Ewertsen C, Carlsen $J$ et al. (2016): Ultrasound elastography is useful for evaluation of liver fibrosis in children: A systematic review. JPG., 63: 389-399. 
9. Hanquinet S, Rougemont A, Courvoisier D et al. (2013): Acoustic radiation force impulse ARFI elastography for the noninvasive diagnosis of liver fibrosis in children. Pediatr Radiol., 43: 545-51.

10. Pinto J, Matos H, Nobre S et al. (2014): Comparison of acoustic radiation force impulse/serum noninvasive markers for fibrosis prediction in liver transplantation. J Pediatr Gastroenterol Nutr., 58: 382-6.

11. Ferraioli G, Lissandrin R, Tinelli C et al. (2016): Liver stiffness assessed by transient elastography[TE] in patients with thalassemia major. Annals of Hepatology, 15(3): 410-417.

12. Tomato W, Yoneda M, Imajo K et al. (2013): Evaluation of the liver fibrosis index calculated by using real-time tissue elastography for the noninvasive assessment of liver fibrosis in chronic liver diseases. Hepatol Res., 43:735-42

13. Tamaki N, Kurosaki M, Matsuda S et al. (2013): Prospective comparison of real-time tissue elastography and serum fibrosis markers for the estimation of liver fibrosis in chronic hepatitis $\mathrm{C}$ patients. Hepatol Res., 13: 1-5.

14. Wang J, Gao L, Shi $X$ et al. (2012): Real-time ultrasonic elastography with a novel quantitative technology for assessment of liver fibrosis in chronic hepatitis B. Eur J Radiol., 81: 31-6.

15. Morikawa H, Fukuda K, Kobayashi S et al. (2011): Real-time elastography[RTE] as a tool for the noninvasive assessment of liver stiffness in patients with chronic hepatitis C. J Gastroenterol., 16: 350-8.

16. Colombo S, Buonocore M, Poggio A et al. (2012): Head-to-head comparison of Transient Elastography (TE), Real-time Tissue Elastography (RTE), and Acoustic Radiation Force Impulse (ARFI) imaging in the diagnosis of liver fibrosis. J Gastroenterol., 47: 461-9.

17. Yada N, Kudo M, Morikawa H et al. (2013): Assessment of Liver fibrosis with real-time tissue elastography[RTE] in chronic viral hepatitis. Oncology, 84: 13-20.

18. Orlacchio A, Bolacchi F, Antonicoli M et al. (2012): Liver elasticity in Non-Alcoholic Steatohepatitis in patients evaluated with Real-Time Elastography (RTE). Ultrasound Med Biol., 38: 537-44.

19. Venturi C, Sempoux C, Bueno J et al. (2012): Novel histologic scoring system for long-term allograft fibrosis after liver transplantation in children. Am J Transplant.

2986-96. 